\title{
Meloidogyne incognita (Kofoid and White, 1919) Chitwood 1949 y Meloidogyne arenaria (Neal 1889) Chitwood 1949: NEMATO DO S DE LAS NUDO SIDADES RADICULARES EN GUAYABA (Psidium guajava L.) C.V. MANZANA EN MONTERIA, CORDOBA
}

\author{
Meloidogyne incognita (Kofoid and White, 1919) Chitwood 1949 y \\ Meloidogyne arenaria (Neal 1889) Chitwood 1949: RO OT- KNOT \\ NEMATO DES IN GUAVA (Psidium guajava L.) \\ C.V. APPLE IN MONTERIA, CORDOBA
}

\author{
Juan D. Jaraba ${ }^{1}$, Zayda Lozano ${ }^{1}$ y Isidro E. Suárez ${ }^{2}$
}

\begin{abstract}
RESU MEN
El presente trabajo tubo como objetivo identificar los nematodos agalladores asociados al cultivo de Guayaba (Psidium guajava L.) en M ontería, Córdoba. Se tomaron muestras de raíces y suelo rizosférico. Las hembras, machos y juveniles de segundo estadío larval (J2) se obtuvieron mediante los métodos de hipoclorito de sodio-fuscina ácida, tamizado centrifugado y eclosión de masas de huevecillos, respectivamente. Se evaluaron variables morfológicas y morfométricas para determinar la especie. Se encontraron dos tipos de patrones perineales: El primer patrón con arco dorsal alto y cuadrado. El estilete de $14 \mu \mathrm{m}$ a $16 \mu \mathrm{m}$, los nódulos anchos y planos. La distancia DGO de $2 \mu \mathrm{m}$ a $4 \mu \mathrm{m}$. Los machos presentaron el disco labial redondeado y cóncavo centralmente. El estilete de $22 \mu \mathrm{m}$ a $25 \mu \mathrm{m}$; nódulos planos y redondeados. La distancia DGO de $2 \mu \mathrm{m}$ a $4.5 \mu \mathrm{m}$. Lo cual es típico de la especie M. incognita. En el segundo tipo de patrón, el arco dorsal es bajo, redondeado. Las estrías se curvan ligeramente hacia las líneas laterales y forman una ondulación, conocida como "hombrera". El estilete midió de 13 $\mu \mathrm{m}$ a $17 \mu \mathrm{m}$, los nódulos son anchos y redondeados en su parte posterior. La distancia DGO de $3 \mu \mathrm{m}$ a $6 \mu \mathrm{m}$. Estas características coinciden con las descritas para M. arenaria. Los resultados permiten el reporte por primera vez de $M$. incognita y $M$. arenaria afectando conjuntamente el cultivo de guayaba en Ia Costa Caribe Colombiana.
\end{abstract}

Palabras claves: Patrones perineales, agallas, estrías

\footnotetext{
${ }^{1}$ Ingeniero Agrónomo, M.Sc. Departamento de Agronomía. Universidad de Córdoba. Email:jjaraban@hotmail.com ${ }^{2}$ Ingeniero Agrónomo Ph.D. Departamento de Agronomía. Universidad de Córdoba
} 


\begin{abstract}
The objective of the present research was to identify at the species level and using morphological and morphometric parameters the root-knot nematode present in guava's crop in Monteria. Samples from roots and ryzospheric soil were taken and the female, male and second stage juvenile larvae (J2) were obtained using the acid sodium hypochlorite-fuscine, mesh centrifuge and egg mass eclossion, respectively. Two types of perineal patterns were observed: the first one with high square dorsal arch, stilettos with 14 to $16 \mu \mathrm{m}$, flat and wide nodules and DGO distance of 2 to $4 \mu \mathrm{m}$. The males showed a rounded lip disc and centrally concave stilettos with 22 to $25 \mu \mathrm{m}$, flat and rounded nodules and a DGO distance of 2 to $4.5 \mu \mathrm{m}$, a typical fact of the $M$. incognita species. The second patterns has low and rounded dorsal arch. The strives form a shoulder, stilettos with 13 to $17 \mu \mathrm{m}$, wide and rounded nodules in the posterior zone, the DGO distance was 3 to $6 \mu \mathrm{m}$. All this characteristics are typical of the $M$. arenaria species. The results showed for first time the simultaneous presence of $M$. incognita and $M$. arenaria attacking guava's crop in the Colombian Caribbean Coast.
\end{abstract}

Key words: Perineal patterns; gill; stria.

\section{INTRO DUCCION}

El Caribe Húmedo Colombiano representa una de las despensas agrícola más importante de Colombia. En ésta región, el Departamento de Córdoba ocupa un lugar privilegiado por la gran variedad de productos agropecuarios que en él se producen. En esta entidad existen $207,577 \mathrm{Ha}$ cultivadas, de las cuales $150,888 \mathrm{Ha}$ se siembran en cultivos transitorios (algodón, maíz, arroz, ajonjolí, sandía, entre otros) y $56,689 \mathrm{Ha}$ en cultivos anuales y permanentes (frutales, hortalizas, entre otros). D entro de las especies frutales cultivo de guayaba (Psidium guajava L.) se considera una fruta con gran potencial ya que el área de siembra se encuentra en constante incremento, al igual que la demanda de ésta como fruta fresca (SADECOR, 2003). Sin embargo en este cultivo se ha visto afectado por una serie de enfermedades causadas por hongos, bacterias, virus y nematodos.

Dentro de los nematodos patógenos de plantas, son de gran importancia económica los que causan agallas en las raíces (Meloidogyne spp. y Nacobbus aberrans Thorne y Allen). Las especies del género Meloidogyne son parásitos obligados de cientos de plantas, entre las que se incluyen monocotiledóneas, dicotiledóneas, herbáceas y plantas forestales que crecen en climas tropicales, subtropical es y templados. El género incluye cerca de 80 especies y once razas, sin embargo, $M$. incognita (Kofoid and White) Chitwood, razas $1,2,3$ y 4; $M$. javanica (Treub, 1885) Chitwood, 1949; M. arenaria (Neal) Chitwood razas 1 y $2 ; \mathrm{M}$. chitwoodi razas 1, 2 y 3 yM. hapla Chitwood, 1949 razas $A$ y $B$, se consideran las especies más comunes e importantes del género y son responsables del $90 \%$ o más del daño a cultivos agrícolas, causado por este tipo de nematodos. Meloidogyne incognita es la especie más ampliamente distribuida, seguida por $M$. javanica, $M$. arenaria, $M$. hapla y M. chitwoodi. Meloidogyne incognita y $M$. javanica se encuentran principalmente en regiones templadas, tropicales y subtropicales del mundo, Meloidogyne hapla y $M$. chitwoodi se distribuyen en regiones de temperaturas frías y $\mathrm{M}$. arenaria se localiza en casi todas las regiones del mundo (Eisenback, 1985; Jepson, 1987; Karssen y Van Hoenselaar, 1998).

En Córdoba, se ha observado la presencia de nudosidades en raíces de tomate (Lycopersicum sculentum L.), berenjena (Solanum melongena L.), guayaba (Psidium guajava L.) y otros cultivos. Sin embargo, no se han realizado estudios tendientes a identificar las especies de este tipo de nematodo. 
Debido a que se desconoce el agente causal de las agallas en las raíces en plantas de guayaba en el Departamento de Córdoba y la existencia de reportes previos de especies de Meloidogyne en éste cultivo (Mosquera et al., 1995; Paéz y Roa, 2003), se planteó el presente trabajo que tuvo como objetivos describir la sintomatología asociado a éste patosistema e identificar la especie de nemato do causante de estas agallas.

\section{MATERIALES Y METODOS}

\section{Descripción de síntomas y muestreo}

Se muestrearon lotes cultivados con guayaba cV Manzana de siete años de edad, en la Finca Risaralda. (Km 22 carretera M ontería Planeta Rica) en el Departamento de Córdoba. En cada lote se tomaron diez submuestras distribuidas al azar de raíces y suelo rizosférico en la zona de goteo de cada planta. Las raíces fueron separadas del suelo y en ambos casos se guardaron en bolsas plásticas, se etiquetaron con el nombre del lote, la finca y el corregimiento. Las muestras fueron procesadas en el laboratorio de Fitopatología de la U niversidad de Córdoba.

\section{O btención de los diferentes estadíos}

Las raíces con agallas se procesaron mediante el método de hipoclorito de sodio-fuscina ácida (Daykin y Hussey, 1985). De éstas se obtuvieron hembras adultas a las que se les realizó un corte perineal de la región anterior. El segundo estadío juvenil (J2) se obtuvo de la eclosión de masas de huevecillos aisladas de agallas en las raíces infectadas asociadas a hembras adultas temporalmente clasificadas mediante cortes perineales. Las muestras de suelos fueron utilizadas para la extracción de los machos mediante el método de tamizadocentrifugado (Hooper, 1986).

\section{Evaluación de características morfológicas y morfométricas}

Se evaluaron las siguientes características morfológicas: forma de las hembras, forma del estilete y de los nódulos en hembras, machos y J2; número de anillos en la región cefálica en machos y J2. En los patrones perineales de las hembras se observó la forma, tipo de arco dorsal, líneas del campo lateral y las estrías. Las características morfométricas evaluadas fueron: Iongitud de las hembras, machos y J2; tamaño del estilete y distancia de la base del estilete a la desembocadura de la glándula dorsal (D.G.O) en hembras, machos y J2. De éstos últimos, se realizaron medidas de la cola, región hialina y longitud del cuerpo. Para examinar estos caracteres se tomaron 25 ejemplares de cada estadío.

\section{RESU LTADOS Y DISC USION}

\section{Descripción de síntomas}

Las plantas de guayaba presentaron defoliación progresiva del ápice de las ramas hacia la base de éstas. En estados finales, la planta pierde completamente el follaje y se observa el síntoma denominado por los agricultores como "variteo", que culmina con la muerte de la planta. Las raíces principales de éstas plantas presentaron consistencia corchosa, se tornan necróticas y agallas coalescidas que la degeneran totalmente. En raíces secundarias, se observan pequeñas agallas redondeadas e individuales. Esta sintomatología coincide con la descrita por Mosquera et al., (1995) en plantas de guayabo afectadas por especies de Meloidogyne. 


\section{Identificación de especies}

\section{Morfología y morfometría de las}

hembras. Las hembras son periformes a redondeadas, hialinas, de $612 \mu \mathrm{m}$ a $815 \mu \mathrm{m}$ x $340 \mu \mathrm{m}$ a $583 \mu \mathrm{m}$ (largo x ancho). Se encontraron dos tipos de patrones perineales: El primer patrón presentó el arco dorsal alto y cuadrado, formado por estrías onduladas, sin campos laterales evidentes (Figura 1). El estilete midió de $14 \mu \mathrm{m}$ a $16 \mu \mathrm{m}(15.3 \mu \mathrm{m} \pm$ $0.8 \mu \mathrm{m})$, presentó el cono curvado hacia la parte dorsal, la columna más ancha en la base y nódulos del estilete anchos y planos. La distancia DGO midió de $2 \mu \mathrm{m}$ a $4 \mu \mathrm{m}(3.2$ $\mu \mathrm{m} \pm 0.5 \mu \mathrm{m}$ ), lo cual es típico de la especie Meloidogyne incognita (Tabla 1).

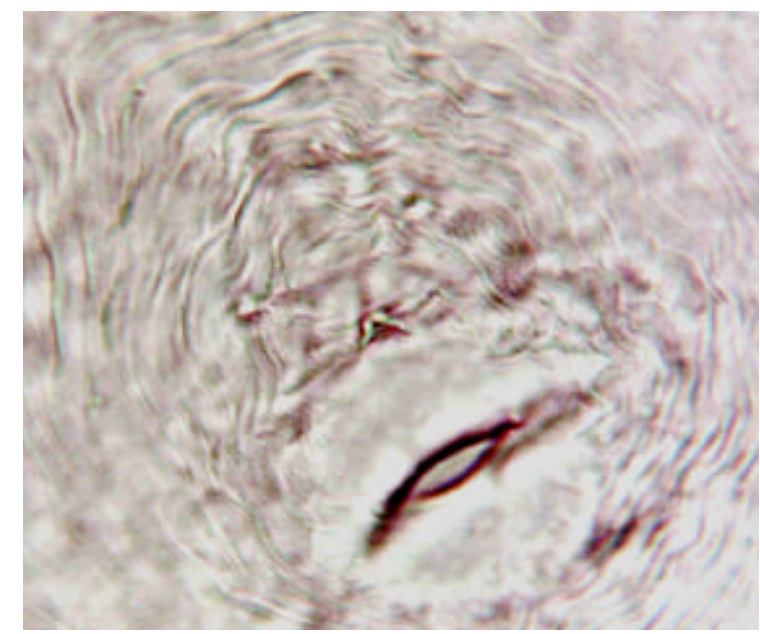

Figura 1. Microfotografía del patrón perineal de Meloidogyne incognita colectados en campos cultivados con Guayaba en Montería, Córdoba.
El segundo tipo de patrón presentó el arco dorsal aplanado o redondeado. Las estrías en el arco se curvan ligeramente hacia las campos laterales y forman una ondulación, conocida como "hombrera". Las estrías son lisas a onduladas (Figura 2). El estilete de las hembras con éste tipo de patrón, midió de $13 \mu \mathrm{m}$ a $17 \mu \mathrm{m}(15.6 \mu \mathrm{m} \pm 0.9 \mu \mathrm{m})$; el cono y la columna son gruesos; la columna incrementa su diámetro hacia la base y los nódulos son anchos y redondeados en su parte posterior. La distancia DGO midió de $3 \mu \mathrm{m}$ a $6 \mu \mathrm{m}(5.2 \mu \mathrm{m} \pm 0.6 \mu \mathrm{m})$. Estas características coinciden con las descritas para Meloidogyne arenaria (Tabla 1).

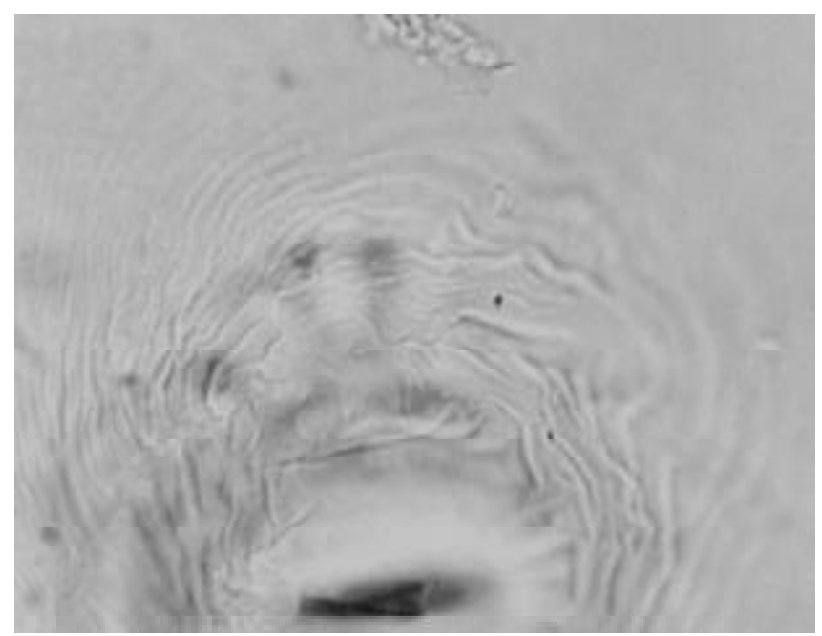

Figura 2. Microfotografía del patrón perineal de Meloidogyne arenaria colectados en campos cultivados con Guayaba en Montería, Córdoba.

Tabla 1. Parámetros morfométricos $(\mu \mathrm{m})$ en M eloidogyne incognita y Meloidogyne arenaria de especimenes aislados en el cultivo de guayaba en Montería, Córdoba.

\begin{tabular}{|l|c|c|c|c|c|c|c|c|c|}
\hline \multirow{2}{*}{$\begin{array}{c}\text { Especies de } \\
\text { Meloidogyne }\end{array}$} & \multicolumn{3}{|c|}{ Hembras } & \multicolumn{4}{c|}{ J u v e n i l e s } & \multicolumn{2}{c|}{ M a c h O S } \\
\cline { 2 - 10 } & Estilete & D G O & Estilete & D G 0 & Cola & $\begin{array}{l}\text { Región } \\
\text { hialina }\end{array}$ & Longitud & Estilete & D G 0 \\
\hline M. incognita & $14-16$ & $2-4$ & $10-14$ & $2-4$ & $50-61$ & $10.6-16$ & $405-560$ & $22-25$ & $2-4.5$ \\
\hline M. arenaria & $13-17$ & $3-6$ & & & & & & & \\
\hline
\end{tabular}


Morfología y morfometría de machos. Los machos encontrados presentaron el disco labial grande y redondeado, cóncavo centralmente y generalmente más al to que los labios medios; la región cefálica con dos o tres anillos incompletos. El estilete presentó una longitud de $22 \mu \mathrm{m}$ a $25 \mu \mathrm{m}(23.8 \mu \mathrm{m} \pm$ $01 \mu \mathrm{m})$ columna cilíndrica y generalmente más angosta cerca de los nódulos de la base; nódulos basales planos y redondeados, ligeramente separados de la columna (Figura 3). La distancia DGO midió de $2 \mu \mathrm{m}$ a 4.5 $\mu \mathrm{m}(3.2 \mu \mathrm{m} \pm 1.1 \mu \mathrm{m})$ (Tabla 1). Estas características concuerdan con las descritas por Eisenback (1985) para los machos de M. incognita. No se encontraron machos para la especie M. arenaria.

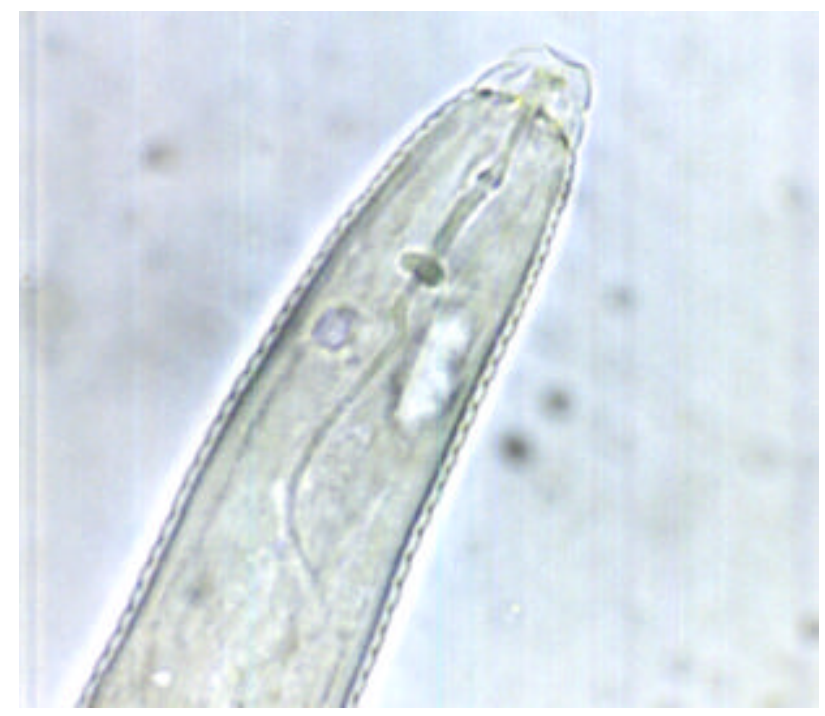

Figura 3. Microfotografía de la región anterior de machos de $M$. incognita colectados en campos cultivados con Guayaba en Montería, Córdoba.

\section{Morfología y morfometría de juveniles del segundo estadío larval (j2)}

Los juveniles midieron $405 \mu \mathrm{m}$ a $560 \mu \mathrm{m}$ (488 $\mu \mathrm{m} \pm 46.7 \mu \mathrm{m})$, son cilíndricos y vermiformes, presentaron el disco labial redondeado, la región cefálica sin anillos. El estilete tiene una longitud de $10 \mu \mathrm{m}$ a $14 \mu \mathrm{m}$ (12.3 $\mu \mathrm{m} \pm 0.9 \mu \mathrm{m})$, el cono y la columna del estile son robustos y de tamaño similar; los nódulos son anchos y posteriormente redondeados. La distancia DGO se localiza de $2 \mu \mathrm{m}$ a $4 \mu \mathrm{m}(3.2 \mu \mathrm{m} \pm 0.9 \mu \mathrm{m})$. La cola mide $50 \mu \mathrm{m}$ a $61 \mu \mathrm{m}(55.8 \mu \mathrm{m} \pm 1.1 \mu \mathrm{m})$, es cónica con anulaciones normales 0 irregulares en la parte terminal. La región hialina mide $10.6 \mu \mathrm{m}$ a $16 \mu \mathrm{m}(14.5 \mu \mathrm{m} \pm$ $0.6 \mu \mathrm{m}$ ) (Tabla 1). Estas características coinciden con las descritas en la literatura para los 2 de M. incognita. No se encontraron juveniles para M. arenaria.

Teniendo en cuenta la descripción de las características morfológicas y morfométricas observados en este estudio, los resultados coinciden con los descritos para M. incognita y $M$. arenaria en raíces de guayaba (O rton, 1972; Eisenback et al., 1981; Eisenback, 1985).

Los resultados de esta investigación coinciden con los encontrados por M osquera et al., (1995) y Páez y Roa (2003), quienes encontraron que el género M eloidogyne fue el más abundante en cultivos de guayaba; sin embargo, ellos no reportan la especie ni la existencia de mezcla de especies.

Resultados similares fueron obtenidos por Villota y Varón (1997) en Palmira (Valle), quienes encontraron que este género de nematodos pudo afectar 23 cultivares de guayaba, incluyendo el de éste estudio. Sin embargo, ellos encontraron sólo la especie Meloidogyne incógnita raza 2.

En Cuba, M. arenaria, M. incógnita, $M$. javanica y $M$. hapla se encontraron parasitando plantaciones de guayaba, siendo la especie $M$. arenaria una de las más difundidas en este cultivo (Cuadra y Quincosa, 1982). Reportes similares hace Sosa-Moss (1985) en México y centro A mérica, donde las especies $M$. incógnita y $M$. arenaria se han encontrado asociada a diversos cultivos, incluyendo guayaba.

Los resultados de estas investigaciones muestran que las especies $M$. incógnita y $M$. 
arenaria reportadas en este trabajo, son frecuentes en este cultivo; sin embargo, en la Costa Caribe Colombiana no se habían reportado éstas. Por lo tanto, podemos reportar por primera vez la mezcla de éstas especies asociadas a este frutal en esta región del país.

La presencia de machos de $M$. incognita y la ausencia de éstos en $M$. arenaria en esta investigación, se ha reportado en otros estudios (Jaraba-Navas et al., 2001). Por consiguiente, el patrón perineal de las hembras, la forma y longitud del estile de las hembras y J2, constituyen caracteres fundamentales utilizados en la clasificación de esta especie. Los resultados fueron corroborados con datos reportados para los mismos caracteres por Eisenbak (1985) y Jepson (1987), y se consideran válidos para la clasificación de especies del género Meloidogyne.

La presencia de la mezcla de $M$. incognita y M. arenaria en guayaba es de gran relevancia por la gran importancia que tiene éste cultivo en el departamento de Córdoba y hace parte de un estudio general de las especies de nematodos agalladores en éste.

\section{CONCLUSIONES Y RECOMENDACIONES}

- La muerte de plantas de guayaba se encuentra relacionada por la presencia de un fuerte ataque de los nematodos agalladores Meloidogyne incognita y Meloidogyne arenaria.

- Se reporta por primera vez la presencia de las especies $M$. incognita y $M$. arenaria causando agallas en guayaba en la Costa Norte Colombiana.

Este tipo de estudios deberá ampliarse a otras especies vegetales con el fin de tener un conocimiento detallado de las especies de Meloidogyne asociados a los cultivos de importancia económica de la región.

\section{BIBLIO G RAFIA}

Cuadra, R. y Quincosa, A. 1982. Comportamiento de diferentes especies de Psidium como patrones para guayabo resistentes a Meloidogyne. Revista Ciencias de la Agricultura 13:19-26

Daykin, M. y H ussey, R. 1985. Staining and histopathological techniques in N ematology. En: Bartker, K.; Carter, C. y Sasser, J. (Ed). An advanced treatise on Meloidogyne. Vol. II, Methodology. North Carolina State U niversity Graphics, p.39-48

Eisenback, J. 1985. Diagnostic characters useful in the identification of the four most common species of rooot-knot nematodes (Meloidogyne spp). En: Bartker, K.; Carter, C. y Sasser, J. (Ed). An advanced treatise on Meloidogyne. Vol. II, M ethodology. North Carolina State University Graphics, p.95-112

Eisenback, J.; Hirschmann, H.; Sasser, J. y Triantaphyllou, A. 1981. A guide to the four most common species of root-knot nematodes (Meloidogyne species). Plant Pathology and Genetics. North Carolina State University and the United States Agency for International D evelopment, Raleigh, p.45-60 
Hooper, D. 1986. Extractión of free-living stages of soil. In: Southey, J. (Ed). Laboratory methods for work with plant and soil nematodes. Ministry of Agriculture Fisheries and Food, Reference Book 402. Her Majesty's Stationery $\mathrm{O}$ ffice. p. 5-30

Jaraba-Navas, J.; Guzmán-Plazola, R.; Caswell-Chen, E.; Zavaleta-M ejía, E. y Del Prado, I. 2001. Especies y razas de Meloidogyne asociadas al cultivo del Tomate (Lycopersicon esculentum Mill.). Tesis M.Sc., Especialista en Fitopatología. Colegio de Postgraduados, Montecillos, p.98

Jepson, S. 1987. Identification of root-knot nematodes (Meloidogyne species). C.A.B. International United Kingdom. Londres, p.265

Karssen, G. y Van Hoerselaar, T. 1998. Revision of the genus Meloidogyne Göldi, 1892 (Nematoda: Heteroderidae) in Europe. Nematologica 44:713-788

Mosquera, A.; Murcia, N. y Varón de Agudelo, F. 1995. Susceptibilidad del guayabo a nematodos fitoparasitos. ASCO LFI INFO RMA 20:71-75
Orton, W. 1972. Meloidogyne javanica. C.I.H. Descriptions of Plant-parasitic Nematodes Set 1, No. 3 . Commonwealth Institute of Helminthology, St. Albins, p.8

Paéz, A. y Roa, N . 2003. Identificación de la nematofauna asociada a cultivares de guayaba (Psidium guajaba L.) en el municipio zona bananera, departamento del Magdalena. ASCO LFI IN FO RMA. 29:27-28.

SADECOR (Secretaría de Agricultura de Córdoba). 2001. Anuario estadístico, p.366-368

Sosa-M oss, C. 1985. Report on the status of Meloidogyne research in México, Central América and the Caribbean countries. En: Bartker, K.; Carter, C. y Sasser, J. (Ed). An advanced treatise on Meloidogyne. Vol. I, Methodology. North Carolina State U niversity Graphics, p.327-346

Villota, F, y Barón, F. 1997. Evaluación de materiales de guayaba (Psidium guajava) por su comportamiento al ataque de Meloidogyne incognita Raza Z. Fitopatología Colombiana 21:31-37 